\title{
Diversidad, abundancia relativa y patrones de actividad de mamíferos medianos y grandes en una selva seca del Istmo de Tehuantepec, Oaxaca, México
}

\author{
Malinalli Cortés-Marcial \& Miguel Briones-Salas \\ Laboratorio de Vertebrados Terrestres (Mastozoología), Centro Interdisciplinario de Investigación para el Desarrollo \\ Integral Regional (CIIDIR-Oaxaca), Instituto Politécnico Nacional. Hornos 1003, Santa Cruz Xoxocotlán. C.P. 71230, \\ México; mali_cor@yahoo.com.mx,mbriones@ipn.mx
}

Recibido 03-II-2014. Corregido 30-VI-2014. Aceptado 29-VII-2014.

\begin{abstract}
Diversity, relative abundance and activity patterns of medium and large mammals in a tropical deciduous forest in the Isthmus of Tehuantepec, Oaxaca, Mexico. The use of camera traps and mammal track search are complementary methods to monitoring species of which is not well documented their natural history, as in the case of medium and large mammals. To ensure its conservation and good management, it is necessary to generate information about the structure of the community and their populations. The objective of the present study was to estimate the diversity, relative abundance and activity patterns of medium and large mammals in a tropical deciduous forest located in the Isthmus of Tehuantepec, Oaxaca, Mexico. Samplings were conducted in three month intervals, from September 2011 to May 2013. We used photographic-sampling and track search, two complementary sampling methods. For photographic-sampling, 12 camera traps were placed covering an area of $60 \mathrm{~km}^{2}$, while for the tracks search a monthly tour of four line-transect surveys of three kilometers length each was undertaken. We obtained a total of 344 pictures with 5292 trap-days total sampling effort; in addition, 187 track records in a total of $144 \mathrm{~km}$. With both methods we registered 21 species of mammals, in 13 families and seven orders, and five species resulted in new records to the area. The diversity index of Shannon-Wiener obtained with the method of tracks was $\mathrm{H}^{\prime}=2.41$, while the most abundant species were Urocyon cinereoargenteus $(\mathrm{IAR}=0.23$ ) and Pecari tajacu $(\mathrm{IAR}=0.20)$. By the method of trap the most abundant species were $P$. tajacu (IAR=2.62) and Nasua narica (IAR=1.28). In terms of patterns of activity P. tajacu, N. narica and Odocoileus virginianus were primarily diurnal species; Canis latrans and Leopardus pardalis did not show preference for any schedule in particular, and Didelphis virginiana and Dasypus novemcinctus preferred to have nocturnal activity. This information can be of help to the creation of programs of management and conservation of mammals of medium and large in the Isthmus of Tehuantepec, Oaxaca, México. Rev. Biol. Trop. 62 (4): 1433-1448. Epub 2014 December 01.
\end{abstract}

Key words: camera traps, tracks, mammals, diversity, patterns of activity.

El Istmo de Tehuantepec, al sur de México, es una de las regiones de mayor relevancia biogeográfica para muchos vertebrados terrestres (Ramamoorthy, 1998), en esta zona se presentan procesos de especiación activa y diferenciación poblacional (Orr, \& Smith, 1998; Peterson, Soberón, \& Sánchez-Cordero, 1999). Se conjuntan flora y fauna propia de las áreas montañosas de clima templado y frío, con especies tropicales de clima cálido y húmedo. Además, posee una elevada riqueza de especies de mamíferos (Briones-Salas, \& Sánchez-Cordero, 2004), aves (Navarro, García-Trejo, Peterson, \& Rodríguez-Contreras, 2004) y mariposas, entre otros grupos más (González, Briones-Salas, \& Alfaro, 2004; Luis, Llorente, Warren, \& Vargas, 2004). Por todas estas razones, esta región es considerada por diferentes instancias nacionales e internacionales de importancia para la conservación 
de la biodiversidad (Arriaga, Espinoza, Aguilar, Martínez, Gómez, \& Loa, 2000; PérezGarcía, Meave, \& Gallardo, 2001; González et al., 2004; García-Marmolejo, Escalona-Segura, \& Van der Wal, 2008).

Pese a su gran diversidad, la región presenta graves problemas de conservación, principalmente sus selvas secas, que son consideradas entre los ecosistemas más amenazados en el Continente Americano (Ceballos, \& García, 1995; Olson et al., 2000; Ceballos, \& Martínez, 2010). En esta región, se han desmontado extensas áreas de selva, que han sido destinadas a la siembra de cultivos, principalmente sorgo y maíz; además, son sembrados con pastos para formar praderas que se emplean para criar ganado vacuno (Hernández, Ellis, \& Gallo, 2013). A pesar de esto, aún existen remanentes de vegetación original, como resultado del interés de conservación que tienen las comunidades indígenas que se asientan en esta región. Dichas comunidades han propuesto el establecimiento de "áreas comunales protegidas", sitios que no cuentan con un decreto oficial y que son establecidas por decisión propia de las comunidades (Ortega et al., 2010; Martin, Camacho, Del Campo, Anta, Chapela, \& González, 2011). En estas selvas habitan una gran variedad de especies de plantas y animales que se encuentran en peligro de desaparecer, debido principalmente a la pérdida de hábitat y a la cacería furtiva y/o de subsistencia (PérezGarcía, Meave, \& Salas, 2010).

Los mamíferos medianos y grandes que habitan los trópicos secos, constituyen comunidades muy ricas, con una gran variedad de grupos tróficos (Ahumada et al., 2011). Tienen una gran importancia en la dinámica y mantenimiento de los ecosistemas (Dirzo \& Miranda, 1991), pueden influir en la regeneración y recuperación de las selvas a través de la dispersión y depredación de semillas de numerosas especies vegetales; además, actúan como depredadores y presas, así como controladores biológicos de insectos (Bolaños, \& Naranjo, 2001; Nakashima, Inoue, Inoue-Murayama, $\&$ Sukor, 2010). Por otro lado, son una fuente importante de recursos naturales primordiales que son utilizados como carne de monte por los pobladores que habitan estas selvas (Robinson \& Bennett, 2000; Naranjo \& Cuarón, 2010).

Es importante resaltar, que, a pesar de que los estudios sobre mamíferos en esta zona (Olguin-Monroy, León, Samper-Palacios, \& Sánchez-Cordero, 2008; Barragán, Lorenzo, Morón, Briones-Salas, \& López, 2010; Lira, Galindo-Leal, \& Briones-Salas, 2012) y en este tipo de vegetación (Ortiz-Martínez, \& RicoGray, 2007; Santos-Moreno, \& Ruíz-Velásquez, 2011) se han incrementado en los últimos años, aún hace falta información sobre su historia natural. Por ello, resulta necesaria la elaboración de estudios donde se documenten los patrones de riqueza, abundancia y distribución de dichas especies dentro de los hábitats para lograr su conservación y aprovechamiento.

Existen diferentes métodos para el estudio de mamíferos medianos y grandes; en algunos casos se requiere la captura y la manipulación de los individuos por medio de trampas (Swan, Di Stefano, Chistie, Steel, \& York, 2014), y en otros casos, existen técnicas no invasivas para detectar a estas especies, como el uso de cámaras-trampa y búsqueda de rastros (Zielinski \& Kucera, 1995; Wilson \& Delahay, 2001). Recientemente las cámaras-trampa han sido utilizadas para monitorear la dinámica poblacional, riqueza de especies, patrones de actividad, uso de hábitat y la abundancia relativa de dichas especies, datos esenciales para la conservación de especies silvestres (Varma, Pittet, \& Jamadagni, 2006; Shek, Chan, \& Wan, 2007; Maffei, Noss, \& Fiorello, 2007; Arispe, Venegas, \& Rumiz, 2008; Lyra-Jorge, Ciocheti, Pivello, \& Meirelles, 2008; Marnewick, Funston, \& Karanth, 2008; Tobler, Carrillo-Percastegui, \& Powell, 2009; Monroy-Vilchis, Zarco-González, Rodríguez-Soto, Soria-Díaz, \& Urios, 2011; Lira \& BrionesSalas, 2012). Sin embargo, con la implementación de las cámaras trampa, los estudios enfocados a la búsqueda de rastros han disminuido considerablemente.

Por otra parte, la técnica de búsqueda de rastros, constituye una herramienta valiosa para estimar la presencia de especies, abundancia 
relativa y el uso y selección del hábitat (Simonetti, \& Huareco, 1999; Carrillo, Wong, \& Cuarón, 2000; Guzman-Lenis, \& Camargo Sanabria, 2004; Michalski \& Peres, 2007; Espartosa, Pinptti, \& Pardini, 2011; Munari, Keller, \& Venticinque, 2011; Aranda, 2012).

Se ha demostrado que estos métodos han sido diseñados para estudiar especies o grupo de especies en particular (Odel, \& Knight, 2001; Swan et al., 2014), por lo tanto varían en su aplicabilidad y éxito de detección para diferentes taxa, por lo que si se desea registrar todas las especies de una comunidad, es necesario utilizar una combinación de técnicas de muestreo (Ryan, Philippi, Leiden, Dorcas, Wigley, \& Gobbons, 2002; Doan, 2003; LyraJorge et al., 2008). En este estudio utilizamos datos obtenidos con dos métodos de detección de mamíferos medianos y grandes comúnmente empleados (cámaras-trampa y búsqueda de rastros), para hacer frente a tres objetivos principales: 1. Estimar su diversidad y compararla con las temporadas lluviosa y seca, 2 . Determinar la abundancia relativa y compararla entre temporadas y 3. Analizar los patrones de actividad de los mamíferos medianos y grandes presentes en una selva baja caducifolia del Istmo de Tehuantepec, Oaxaca, México. Los datos generados de estos tres objetivos serán muy importantes para las comunidades indígenas que se asientan en el área de estudio para atender preguntas de investigación y manejo de recursos faunísticos que ayuden a su conocimiento, uso y protección.

\section{MATERIALES Y MÉTODOS}

Área de estudio: Se localiza dentro del Área Comunal Protegida llamada Ojo de Agua Tolistoque y Sierra Tolistoque, en el distrito de Juchitán, en la Planicie Costera de Tehuantepec, Oaxaca, México, a una altitud entre 100 y $400 \mathrm{msnm}$, dentro de las coordenadas $16^{\circ} 38^{\prime}$ a $16^{\circ} 30^{\prime} \mathrm{N}-94^{\circ} 55^{\prime}$ a $94^{\circ} 50^{\prime} \mathrm{W}$ (Ortega et al., 2010; Ortíz-Pérez, Hernández, \& Figueroa, 2004). El clima es cálido subhúmedo con lluvias en verano $\left(\mathrm{Aw}_{0}\right)$, cuenta con una marcada temporada de sequía entre noviembre a junio y lluvias de julio a octubre, con una precipitación total anual de $932.2 \mathrm{~mm}$. La temperatura promedio anual es de $27.6^{\circ} \mathrm{C}$. El tipo de vegetación dominante es selva baja caducifolia (bosque tropical caducifolio sensu Rzedowski, 1978), donde predominan especies de Fabaceae (e.g. Lonchocarpus emarginatus y Myrospermum frutescens) y Mimosaceae (e.g. Acacia picachensis y Havardia campylacantha) (García, 1988; Ortega et al., 2010; Pérez-García, Meave, \& Gallardo, 2001).

Registro de mamíferos medianos y grandes: El muestreo fotográfico se desarrolló desde septiembre 2011 a agosto 2013, con cuatro periodos de muestreo con un promedio de 110 días cada uno, cubriendo un total de 216 días durante la temporada seca y 225 días durante la lluviosa.

Se ubicaron 12 estaciones de muestreo que fueron georreferenciadas con un geoposicionador (Garmin etrex ${ }^{\circledR}$ ). Durante cada periodo, se colocaron 12 cámaras trampa marca Cuddeback ${ }^{\circledR}$, a una altura entre 30 y $50 \mathrm{~cm}$ del nivel del suelo, en veredas naturales, caminos abandonados, cañadas y arroyos secos; con una separación aproximada de $2 \mathrm{~km}$ de distancia entre cada una, cubriendo un área de $60 \mathrm{Km}^{2}$. El circuito de la cámara-trampa fue programado para permanecer activo durante 24 horas y con un retraso mínimo de 60 segundos entre cada disparo, en cada fotografía se imprimió la fecha y la hora. Todas las estaciones de muestreo fueron revisadas mensualmente. El esfuerzo de muestreo se obtuvo multiplicando el número total de cámaras-trampa por el total de días que permanecieron activas (Medellín et al., 2006). Los registros fotográficos se prepararon en formato de ficha digital y se depositaron en la Colección de Mamíferos (OAX.MA.026.0497) del Centro Interdisciplinario de Investigación para el Desarrollo Integral Regional, Unidad Oaxaca (CIIDIR, OAX), del Instituto Politécnico Nacional (IPN). Los individuos fotografiados fueron identificados siguiendo la literatura especializada (Reid, 1997; Ceballos, \& Oliva, 2005; Aranda, 2012). 
La búsqueda de rastros (heces fecales y huellas), se realizó desde septiembre 2011 a julio 2013, con 12 periodos de muestreo, seis durante la temporada seca y seis durante la temporada lluviosa. En cada periodo se recorrieron cuatro transectos lineales de $3 \mathrm{~km}$ de longitud cada uno, distribuidos al azar dentro del área de estudio. Para cada registro se ubicó la localidad con un geoposicionador (Garmin etrex $\left.{ }^{\circledR}\right)$, fecha, hora, altitud y medidas de los rastros (ancho, largo y diámetro).

Las heces fecales, se recolectaron en bolsas de papel y se secaron a la intemperie. Para asegurar la correcta identificación de la especie, se consultaron guías especializadas (Aranda, 2012), y se buscaron huellas asociadas a ellas. Las huellas fueron recolectadas a través de la elaboración de moldes de yesos de fraguado rápido (Aranda, 2012) y se identificaron a nivel de especie de acuerdo con Hall (1981); Emmons \& Feer (1990); Reid (1997); Ceballos, \& Oliva (2005) y Aranda (2012). Para ambos casos y para asegurar la correcta identificación, se consultó el material de referencia depositado en la Colección de Mamíferos (OAX.MA.026.0497) del CIIDIR, OAX, del IPN. Una vez identificados los rastros, los moldes de yeso de las huellas fueron depositadas en la colección antes citada. El esfuerzo de muestreo de rastros se obtuvo como el número total de rastros por distancia recorrida.

El gremio trófico de las especies registradas se clasificó en herbívoros, insectívoros, frugívoros, carnívoros y omnívoros, de acuerdo a Ceballos \& Oliva (2005). Para identificar especies de nuevo registro en el municipio de Juchitán, Oaxaca, se revisaron los registros previos de los mamíferos medianos y grandes para los diferentes distritos de Oaxaca, México (Goodwin, 1969; Hall, 1981; Briones-Salas, \& Sánchez-Cordero, 2004). Finalmente, para conocer la situación de riesgo de los mamíferos registrados se consultó la norma oficial mexicana: NOM-059-ECOL-2010 (SEMARNAT 2010) y la lista roja de la Unión Internacional para la Conservación de la Naturaleza (UICN 2011).
Se obtuvo el número de especies registradas por cada uno de los métodos. Se utilizaron dos modelos asintóticos de acumulación de especies, para determinar el éxito en el esfuerzo de captura: Clench y Dependencia lineal (Moreno, \& Halffter, 2000), seleccionando al final el más adecuado con el criterio de máxima verosimilitud por medio del programa Species accumulation v. Beta (http://www.cimat.mx/ en/Descargas_de_software). Los datos se aleatorizaron 100 veces, se calculó el valor de la asíntota y en su caso el esfuerzo adicional necesario para registrar el $95 \%$ del valor asintótico predicho por el modelo seleccionado.

La diversidad alfa $(\alpha)$ se estimó para cada método, de acuerdo al índice de ShannonWiener. Para las temporadas lluviosa y seca, el índice se comparó por medio de la prueba $t$ modificada de Hutchinson (Moreno, 2001). Además de esto, se estimó la dominancia con el índice de Berger-Parker (Whittaker, 1972), que considera la importancia proporcional de las especies más abundantes y, el índice de equidad de Pielou (Magurran, 1988), que mide la proporción de la diversidad observada con relación a la máxima diversidad esperada (Moreno, 2001).

La abundancia de las especies se estimó sumando el número de registros para cada especie en ambos métodos. La abundancia relativa de las especies con el método de fototrampeo se determinó, como el número de registros fotográficos independientes por cada 100 díastrampa. Un registro independiente considera: fotografías consecutivas de diferentes individuos, fotografías consecutivas de individuos de la misma especie separadas por más de 24 horas y fotografías no consecutivas de individuos de la misma especie. Para las especies gregarias, el número de registros independientes fue igual al número de individuos observados en una fotografía (O'Brien, Kinnaird, \& Wibisono, 2003; Yasuda, 2004; Medellín et al., 2006; Monroy-Vilchis, Zarco-González, Rodríguez-Soto, Soria-Díaz, \& Urios, 2011; Lira-Torres, \& Briones-Salas 2012). La abundancia relativa para cada especie con el método de rastros se estimó al dividir el número de 
rastros encontrados (huellas) por especie, por la distancia recorrida (Carrillo et al., 2000).

Para ambos métodos, se obtuvo la abundancia relativa por temporada y se realizó una prueba de Mann-Whitney para determinar diferencias significativas entre temporadas (Zar, 1999).

Se determinó el patrón de actividad general y por temporada para aquellas especies que contaran con al menos 11 fotografías independientes (Maffei, Cuellar, \& Noss, 2002; Monroy-Vilchis et al., 2011). Las imágenes obtenidas se agruparon en intervalos de dos horas y el patrón de actividad se clasificó en diurnas (de las 8:00 a las 18:00 horas), nocturnas (de las 20:00 a las 06:00 horas); crepuscular (matutino, entre las 6:00 y las 8:00 horas y vespertino entre las 18:00 y las 20:00 horas), las especies que no mostraron un patrón claro se clasificaron como catemerales (Maffei et al., 2002; Monroy-Vilchis et al., 2011; Bernard et al., 2013).

\section{RESULTADOS}

Se registraron 21 especies de mamíferos medianos y grandes con ambos métodos, que corresponden a 13 familias y siete órdenes. El orden mejor representado fue Carnívora, con cuatro familias y 11 especies (Cuadro 1).

Con el método de fototrampeo se registraron 18 especies en 344 fotografías, 322 de éstas independientes, con un esfuerzo de muestreo de 5292 días-trampa. Durante la temporada seca se registraron 14 especies en 232 fotografías, mientras que en la temporada lluviosa se registraron 15 especies en 112 fotografías (Cuadro 1).

Por medio del método de rastros se registraron 16 especies, se obtuvieron 187 registros en $144 \mathrm{~km}$ recorridos. Para la temporada seca, se registraron 13 especies en 72 registros, mientras que para la temporada lluviosa se registraron 16 especies en 115 registros (Cuadro 1).

Del total de especies, cinco se registraron únicamente con el método de fototrampeo (Tamandua mexicana, Panthera onca, $S$. pygmaea, Mephitis macroura y D. mexicana), mientras que tres especies se registraron solamente con el método de rastros (Philander opossum, B. astutus y Coendou mexicanus).

El modelo de acumulación de especies que mejor se ajustó a los datos para ambos métodos fue el de Clench, dicho modelo sugiere que en el área de estudio no se ha llegado a la asíntota, el modelo predice que se pueden encontrar 23 especies con el método de fototrampeo $(\mathrm{a}=3.91$ y $b=0.16)$ y 17 especies con el método de rastros $(a=15.27$ y $b=0.87)$, por lo que se tiene una representatividad del $78 \%$ y $92 \%$ de las especies para cada método.

La diversidad de mamíferos medianos y grandes estimada con el índice de ShannonWiener mediante el método de fototrampeo fue de $\mathrm{H}^{\prime}=1.89$, con una diversidad máxima de $H^{\prime} \max =2.89$. Para las diferentes temporadas se observó una mayor diversidad durante la temporada lluviosa $(\mathrm{t}=4.49$, g.l. $=193, \mathrm{p}=0.05)$. El índice de Berger-Parker indica que la comunidad de mamíferos presentó un grado de dominancia medio durante todo el estudio $(D=0.41)$, al igual que para las temporadas seca $(\mathrm{D}=0.39)$ y lluviosa $(\mathrm{D}=0.45)$. El índice de equidad de Pielou fue de $\mathrm{J}=0.65$ durante todo el estudio, en tanto que para la temporada lluviosa fue más alto que para la temporada seca (Cuadro 2).

Mediante el método de rastros, el índice de diversidad fue de $\mathrm{H}^{\prime}=2.41$, con una diversidad máxima de $\mathrm{H}^{\prime} \max =2.77$. De acuerdo a la época del año, existieron diferencias significativas, se registró una diversidad mayor durante la temporada de lluvias $(\mathrm{t}=2.21$, g.l. $=72, \mathrm{p}=0.05)$. La equidad de Pielou fue alta para todo el estudio $\left(\mathrm{J}^{\prime}=0.86\right)$, al igual que para las temporadas. El índice de Berger-Parker indica una dominancia baja para todo el estudio $(\mathrm{D}=0.18)$, mientras que para la temporada seca fue mayor $(\mathrm{D}=0.31)$ (Cuadro 2).

Las especies con mayor abundancia relativa por el método de fototrampeo fueron P. taja$c u(\mathrm{IAR}=2.62, \mathrm{n}=139), N$. narica $(\mathrm{IAR}=1.28$, $\mathrm{n}=68$ ), mientras que siete especies fueron menos abundantes (IAR $=0.019, \mathrm{n}=1$ ); para ambas temporadas $P$. tajacu fue la especie más abundante con un $\mathrm{IAR}=3.47, \mathrm{n}=90$ en secas $\mathrm{y}$ 


\section{CUADRO 1}

Mamíferos medianos y grandes registrados en la selva seca en el Istmo de Tehuantepec, Oaxaca, México. Arreglo sistemático de acuerdo a Ramírez-Pulido et al. 2005

TABLE 1

Medium and large mammals recorded in a tropical deciduous forest located in the Isthmus of Tehuantepec, Oaxaca, Mexico. Taxonomic arrangement proposed by Ramírez-Pulido et al. 2005

\begin{tabular}{|c|c|c|c|c|c|c|c|c|c|c|c|c|c|c|c|}
\hline \multirow{3}{*}{ ORDEN / Familia / Especie } & \multirow{3}{*}{$\begin{array}{l}\text { Grem. } \\
\text { Tróf. }\end{array}$} & \multicolumn{6}{|c|}{ Fototrampeo } & \multicolumn{6}{|c|}{ Rastros } & \multirow{3}{*}{$\begin{array}{l}\text { NOM-059- } \\
\text { SEMARNAT- } \\
2010\end{array}$} & \multirow{3}{*}{ UICN } \\
\hline & & \multicolumn{2}{|c|}{ Secas } & \multicolumn{2}{|c|}{ Lluvias } & \multicolumn{2}{|c|}{ Total } & \multicolumn{2}{|c|}{ Secas } & \multicolumn{2}{|c|}{ Lluvias } & \multicolumn{2}{|c|}{ Total } & & \\
\hline & & $\mathrm{N}$ reg & $\mathrm{Ar}$ & $\mathrm{N}$ reg & $\mathrm{Ar}$ & N reg & $\mathrm{Ar}$ & $\mathrm{N}$ reg & $\mathrm{Ar}$ & $\mathrm{N}$ reg & $\mathrm{Ar}$ & $\mathrm{N}$ reg & $\mathrm{Ar}$ & & \\
\hline \multicolumn{16}{|l|}{ DIDELPHIMORPHIA } \\
\hline \multicolumn{16}{|l|}{ Didelphidae } \\
\hline Philander opossum & omniv & 0 & 0 & 0 & 0 & 0 & 0 & 0 & 0 & 1 & 0.014 & 1 & 0.007 & & \\
\hline Didelphis virginiana & omniv & 25 & 0.965 & 3 & 0.111 & 28 & 0.529 & 10 & 0.139 & 9 & 0.125 & 19 & 0.132 & & \\
\hline \multicolumn{16}{|l|}{ CINGULATA } \\
\hline \multicolumn{16}{|l|}{ Dasypodidae } \\
\hline Dasypus novemcinctus & insec & 18 & 0.694 & 9 & 0.333 & 27 & 0.510 & 6 & 0.083 & 12 & 0.167 & 18 & 0.125 & & \\
\hline \multicolumn{16}{|l|}{ PILOSA } \\
\hline \multicolumn{16}{|l|}{ Myrmecophagidae } \\
\hline Tamandua mexicana & insec & 1 & 0.039 & 0 & 0 & 1 & 0.019 & 0 & 0 & 0 & 0 & 0 & 0 & $\mathrm{P}$ & \\
\hline CARNIVORA & & & & & & & & & & & & & & & \\
\hline Canidae & & & & & & & & & & & & & & & \\
\hline Canis latrans & carniv & 22 & 0.849 & 10 & 0.370 & 32 & 0.605 & 6 & 0.083 & 9 & 0.125 & 15 & 0.104 & & \\
\hline Urocyon cinereoargenteus & omniv & 0 & 0 & 1 & 0.037 & 1 & 0.019 & 22 & 0.306 & 11 & 0.153 & 33 & 0.229 & & \\
\hline Felidae & & & & & & & & & & & & & & & \\
\hline Panthera onca & carniv & 0 & 0 & 1 & 0.037 & 1 & 0.019 & 0 & 0 & 0 & 0 & 0 & 0 & $\mathrm{P}$ & NT \\
\hline Puma concolor & carniv & 3 & 0.116 & 3 & 0.111 & 6 & 0.113 & 2 & 0.028 & 1 & 0.014 & 3 & 0.021 & & \\
\hline Leopardus pardalis & carnív & 11 & 0.424 & 6 & 0.222 & 17 & 0.321 & 4 & 0.056 & 4 & 0.056 & 8 & 0.056 & $P$ & \\
\hline Mephitidae & & & & & & & & & & & & & & & \\
\hline Conepatus leuconotus & insec & 1 & 0.039 & 2 & 0.074 & 3 & 0.057 & 2 & 0.028 & 3 & 0.042 & 5 & 0.035 & & \\
\hline Spilogale pygmaea & insec & 0 & 0.000 & 1 & 0.037 & 1 & 0.019 & 0 & 0 & 0 & 0 & 0 & 0 & & \\
\hline Mephitis macroura & insec & 1 & 0.039 & 0 & 0 & 1 & 0.019 & 0 & 0 & 0 & 0 & 0 & 0 & & \\
\hline Procyonidae & & & & & & & & & & & & & & & \\
\hline Nasua narica & omniv & 49 & 1.890 & 19 & 0.704 & 68 & 1.285 & 2 & 0.028 & 3 & 0.042 & 5 & 0.035 & & \\
\hline Bassariscus astutus & omniv & 0 & 0 & 0 & 0 & 0 & 0 & 1 & 0.014 & 1 & 0.014 & 2 & 0.014 & & \\
\hline Procyon lotor & omniv & 0 & 0 & 1 & 0.037 & 1 & 0.019 & 0 & 0 & 5 & 0.069 & 5 & 0.035 & & \\
\hline ARTIODACTYLA & & & & & & & & & & & & & & & \\
\hline Tayassuidae & & & & & & & & & & & & & & & \\
\hline Pecari tajacu & herbiv & 90 & 3.472 & 49 & 1.815 & 139 & 2.627 & 5 & 0.069 & 24 & 0.333 & 29 & 0.201 & & \\
\hline Cervidae & & & & & & & & & & & & & & & \\
\hline Odocoileus virginianus & herbiv & 4 & 0.154 & 5 & 0.185 & 9 & 0.170 & 5 & 0.069 & 22 & 0.306 & 27 & 0.188 & & \\
\hline RODENTIA & & & & & & & & & & & & & & & \\
\hline Sciuridae & & & & & & & & & & & & & & & \\
\hline Sciurus aureogaster & herbiv & 1 & 0.039 & 1 & 0.037 & 2 & 0.038 & 6 & 0.083 & 2 & 0.028 & 8 & 0.056 & & \\
\hline Agoutidae & & & & & & & & & & & & & & & \\
\hline Dasyprocta mexicana & frugiv & 1 & 0.039 & 0 & 0 & 1 & 0.019 & 0 & 0 & 0 & 0 & 0 & 0 & & \\
\hline Eretizontidae & & & & & & & & & & & & & & & \\
\hline Coendou mexicanus & omniv & 0 & 0 & 0 & 0 & 0 & 0 & 0 & 0 & 2 & 0.028 & 2 & 0.014 & $\mathrm{~A}$ & \\
\hline LAGOMORPHA & & & & & & & & & & & & & & & \\
\hline Leporidae & & & & & & & & & & & & & & & \\
\hline Sylvilagus floridanus & herbiv & 5 & 0.193 & 1 & 0.037 & 6 & 0.113 & 1 & 0.014 & 6 & 0.083 & 7 & 0.049 & & \\
\hline Tot. Registros & & 232 & & 112 & & 344 & & 72 & & 115 & & 187 & & & \\
\hline Tot. especies & & 14 & & 15 & & 18 & & 13 & & 16 & & 16 & & & \\
\hline
\end{tabular}

$\mathrm{N}$ reg=Número de registros independientes obtenidos para cada método, Ar=Abundancia relativa. Gremios tróficos: omniv=Omnívoro, insec=Insectívoro, carniv=Carnívoro, frugiv=Frugívoro, herbiv=Herbívoro. NOM-059SEMARNAT-2010: A=Amenazado, $\mathrm{P}=$ En peligro de extinción. UICN: LC=Preocupación menor, NT=Casi amenazado.

$\mathrm{N}$ reg=Number of independent records by each method, Ra=Relative abundance. Trophic guild: omniv=Omnivore, insec=Insectivore, carniv=Carnivore, frugiv=Frugivore, herbiv=Herbivore. NOM-059-SEMARNAT-2010: T=Threatened, E=Endangered. UICN: LC=Least concern; NT=Near Threatened. 
CUADRO 2

Especies registradas en la selva seca del Istmo de Tehuantepec, Oaxaca, México

TABLE 2

Species recorded in a tropical deciduous forest in the Isthmus of Tehuantepec, Oaxaca, Mexico

\begin{tabular}{|c|c|c|c|c|c|c|}
\hline & \multicolumn{3}{|c|}{ Fototrampeo } & \multicolumn{3}{|c|}{ Rastros (km recorridos) } \\
\hline & Secas & Lluvias & Total & Secas & Lluvias & Total \\
\hline Esfuerzo de muestreo & 2592 días-trampa & 2700 días-trampa & 5292 días-trampa & $72 \mathrm{~km}$ & $72 \mathrm{~km}$ & $144 \mathrm{~km}$ \\
\hline Registros independientes & 232 & 112 & 344 & 72 & 115 & 187 \\
\hline Especies registradas & 14 & 15 & 18 & 13 & 16 & 16 \\
\hline Especies exclusivas & 3 & 4 & 4 & 3 & 0 & 3 \\
\hline Diversidad Shannon-Wiener $\left(\mathrm{H}^{\prime}\right)$ & 1.83 & 1.90 & 1.89 & 2.21 & 2.37 & 2.41 \\
\hline $\mathrm{H}^{\prime} \max$ & 2.64 & 2.71 & 2.89 & 2.56 & 2.77 & 2.77 \\
\hline Dominancia $(D)$ & 0.39 & 0.45 & 0.41 & 0.31 & 0.21 & 0.18 \\
\hline Equidad $\left(J^{\prime}\right)$ & 0.69 & 0.70 & 0.65 & 0.86 & 0.85 & 0.86 \\
\hline
\end{tabular}

$\mathrm{IAR}=1.81, \mathrm{n}=49$ en lluvias. Entre ambas temporadas se observaron diferencias significativas $(\mathrm{U}=82.5, \mathrm{p}=0.32)$.

De acuerdo al método de rastros, las especies más abundantes fueron $U$. cinereoargenteus $(\mathrm{IAR}=0.23, \mathrm{n}=33)$ y $P$. tajacu $(\mathrm{IAR}=0.20$, n=29), mientras que la menos abundante fue $P$. opossum ( $\mathrm{IAR}=0.007, \mathrm{n}=1)$. Durante la temporada seca, $U$. cinereoargenteus fue la especie más abundante ( $\mathrm{IAR}=0.30, \mathrm{n}=22)$, y para la temporada lluviosa $P$. tajacu $(\mathrm{IAR}=0.33$, $\mathrm{n}=24$ ) (Cuadro 1). De acuerdo a la temporalidad existieron diferencias significativas ( $\mathrm{U}=94.5, \mathrm{p}=0.69$ ).

Entre las especies con mayor número de registros, tres presentaron mayor actividad en horarios diurnos: P. tajacu (entre 06:00 y 12:00h), N. narica (entre 8:00 y 16:00h) y O. virginianus (entre 08:00 y 20:00h). Dos especies fueron nocturnas $D$. virginiana (entre 20:00 y 04:00h) y $D$. novemcinctus (entre 20:00 y 06:00h). Finalmente, C. latrans y L. pardalis no presentaron preferencia hacia algún horario en particular, por lo que se les consideró catemerales o generalistas (Fig. 1, Cuadro 3). Se pudo obtener el patrón de actividad por temporadas sólo de cuatro especies, $P$. tajacu, $N$. narica, C. latrans y D. novemcinctus, los patrones de actividad no fueron diferentes a los anuales (Cuadro 3).

De las 21 especies registradas con ambos métodos, los omnívoros estuvieron representados por siete especies, los insectívoros por cinco, los carnívoros y herbívoros por cuatro especies cada uno y una espefie fue frugívora (Cuadro 2).

El $61.90 \%$ de las especies registradas, se encuentran bajo algún criterio de conservación. Cuatro especies se reportan dentro de la legislación mexicana (NOM-059-SEMARNAT 2010): T. mexicana, $P$. onca, $L$. pardalis y $C$. mexicanus, las primeras tres en peligro de extinción y la última como amenazada. $P$. onca se encuentra dentro de la lista internacional (UICN 2011) como casi amenazado.

\section{DISCUSIÓN}

El uso de métodos complementarios puede proporcionar información valiosa en estudios sobre los patrones generales de riqueza de especies, abundancia relativa y diversidad. Varios estudios han demostrado la eficiencia de complementar estos trabajos con diversos métodos (Gaidet-Drapier, Frtz, Bogarel, Renaud, Poilecot, \& Chardonnet 2006; Lyra-Jorge et al., 2008; Swan et al., 2014). Para este caso, se utilizaron los métodos de fototrampeo y el de búsqueda de rastros, se logró registrar un gran número de especies para la zona $(\mathrm{n}=21)$. La mayor parte de estas $(n=13)$ se registraron con ambos métodos, mientras que con el método de fototrampeo se registraron exclusivamente 


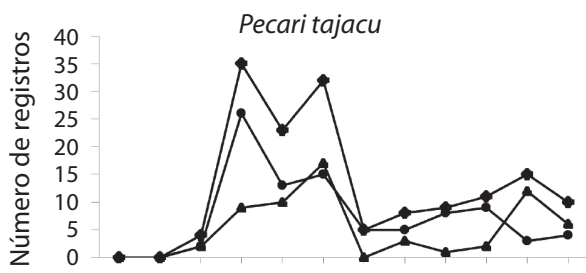

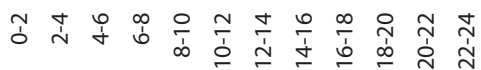

- Total - Hecas . Lluvias
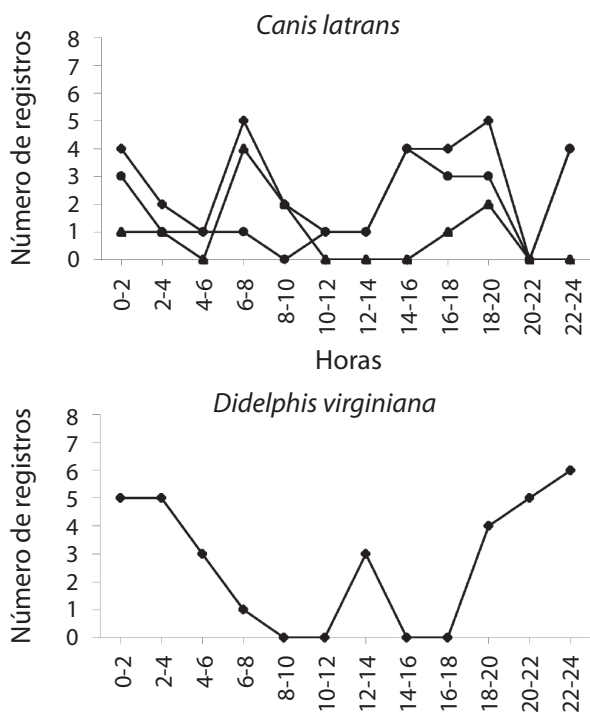

Horas

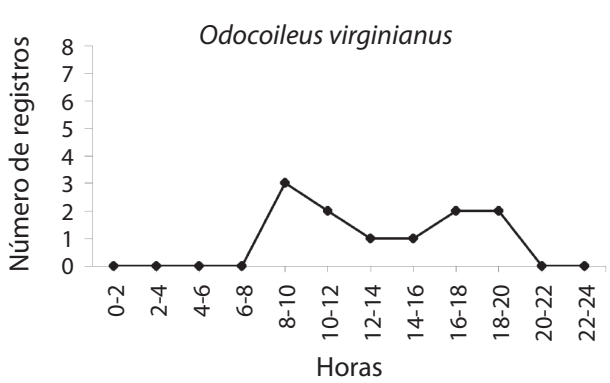

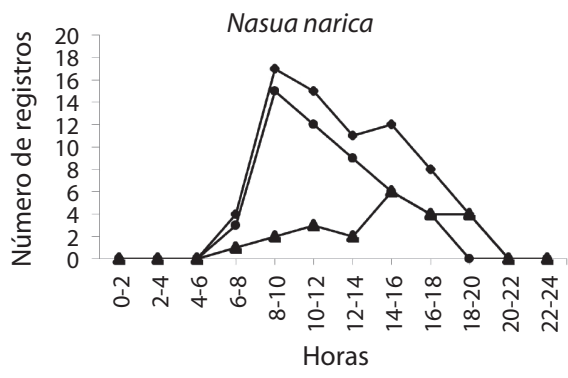

Dasypus novemcinctus

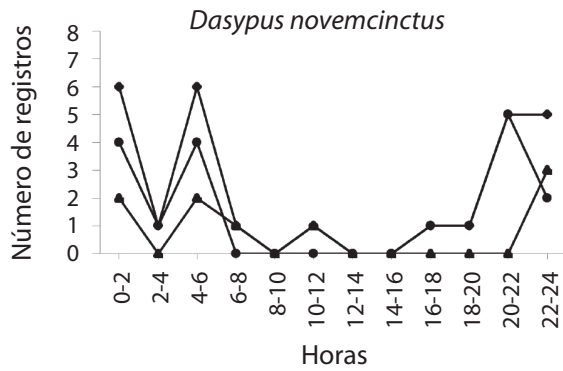

Leopardus pardalis

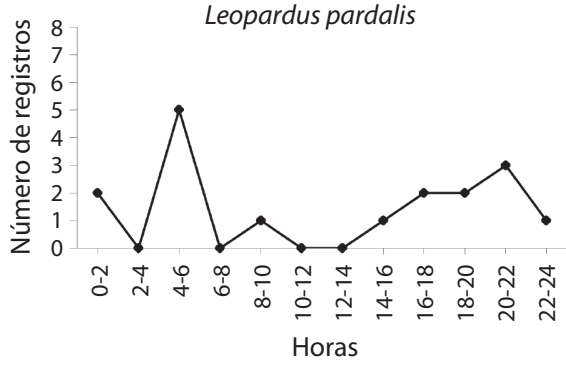

Horas

Fig. 1. Patrones de actividad de mamíferos medianos y grandes en la selva baja caducifolia del Istmo de Tehuantepec, Juchitán, Oaxaca, México.

Fig. 1. Activity patterns of medium and large mammals in a tropical deciduous forest located in the Isthmus of Tehuantepec, Oaxaca, Mexico.

cinco especies y tres más, exclusivamente con el método de rastros.

La riqueza de especies de mamíferos medianos y grandes fue mayor a la registrada en selvas similares en el estado de Oaxaca, México, donde los valores van de 15 a 19 especies (Santos-Moreno, \& Ruíz-Velásquez, 2011; Pérez-Irineo, \& Santos-Moreno, 2012).

Sin embargo, la riqueza encontrada en el sitio de estudio es menor a la registrada en otros sitios de México. Por ejemplo, en Salina Cruz, Oaxaca, se reportan 30 especies de 


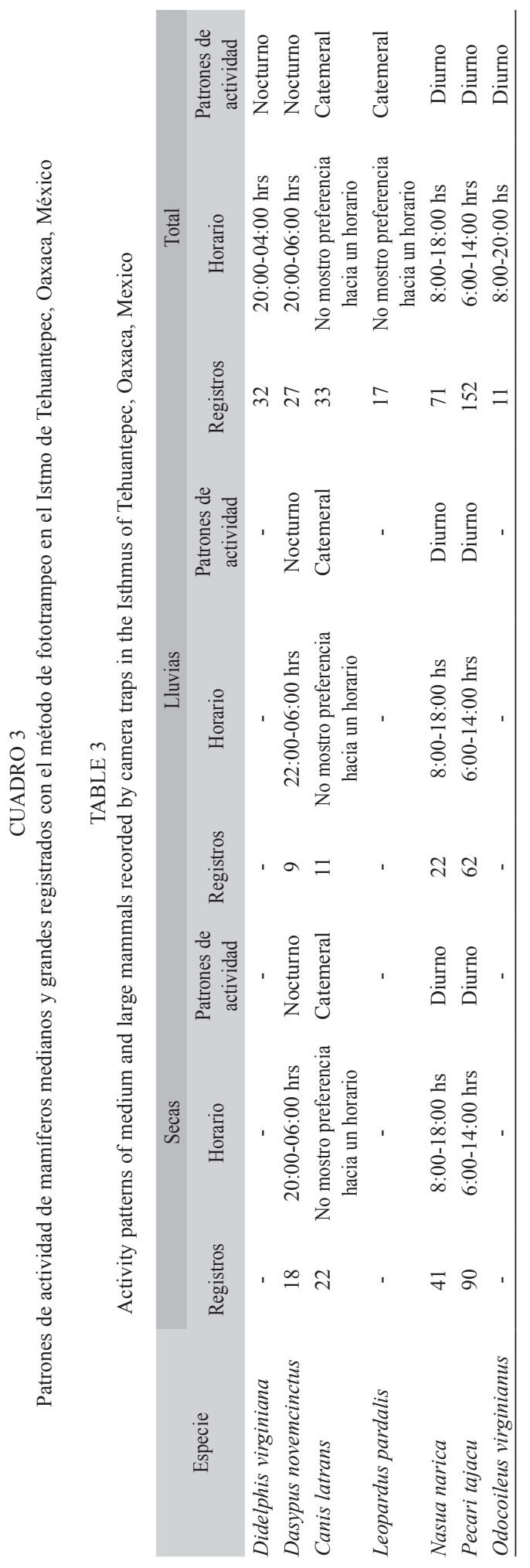

mamíferos medianos y grandes (Cervantes, \& Yepez, 1995); mientras que en Cerro de la Tuza y Parque Nacional Chacahua, en la costa de Oaxaca, se registran 23 especies (BuenrostroSilva, Gutiérrez, \& García-Grajales, 2012). Habría que aclarar que estos trabajos se realizaron en sitios que además de bosque tropical caducifolio, tienen otros tipos de vegetación, que pueden proveer distintos nichos ecológicos y pueden ser ocupados por más especies.

En este estudio, se reporta por primera vez a Dasyprocta mexicana en una selva baja caducifolia, a 400msnm, dicha especie sólo había sido reportada en selva alta y mediana perennifolia y subperennifolia; el registro más cercano para esta especie se encuentra en una selva alta perennifolia en Los Chimalapas, Oax., México, a $72 \mathrm{~km}$ al noreste del sitio de estudio (LiraTorres, \& Briones-Salas, 2012).

El modelo de acumulación de especies de Clench sugiere que aún existen cinco especies por registrar por medio del fototrampeo y una especie por medio de la búsqueda de rastros. En un estudio anterior realizado en el sitio (CortésMarcial, 2009), se reportaron por medio de rastros y observación directa tres especies más, Puma yagouaroundi, Ateles geoffroyi y Mustela frenata. Además es posible que en la zona se distribuya, Didelphis marsupialis y Potos flavus, las cuales fueron reportadas, en una selva baja caducifolia de la región de Mena Nizanda, localizada aproximadamente a 11 kilómetros del sitio de estudio (Santos-Moreno, \& RuízVelásquez, 2011).

Destaca la presencia de $P$. concolor y $P$. onca que requiere de grandes extensiones de hábitat y suficiente alimento disponible para su sobrevivencia. Por otra parte, también sobresale la presencia del zorrillo pigmeo S. pygmaea, especie endémica de México (Ávila, \& Medellín, 2005).

Los valores de diversidad registrados para la zona son más bajos que los reportados por Cortés-Marcial (2009) $\left(\mathrm{H}^{\prime}=2.3\right)$ en una porción del sitio de estudio, esto es debido a que en el estudio previo, las abundancias fueron similares para la mayoría de las especies, mientras que en este estudio se registraron dos especies 
P. tajacu y $N$. narica como muy abundantes, mientras que el resto presentaron abundancias muy bajas, esto pudo comprobarse con los índices de equidad y dominancia.

En contraste, la diversidad resultó mayor que en una selva subcaducifolia en Tuxtepec, Oaxaca $\left(\mathrm{H}^{\prime}=0.89\right)$ (Pérez-Irineo, \& SantosMoreno, 2012), debido a que la zona está siendo transformada a cultivos de caña de azúcar y áreas de potrero.

Las especies más abundantes durante el estudio fueron $P$. tajacu y N. narica, posiblemente como resultado del comportamiento de ambas especies, ya que las hembras adultas y los individuos jóvenes forman grupos sociales (Cooper, Waser, Gopurenko, Hellgren, Gabor, \& Dewoody, 2010; Logan, \& Longino, 2013). Los resultados de abundancia relativa coinciden con lo reportado por Monroy-Vilchis et al. (2011) y Pérez-Irineo, \& Santos Moreno (2012), donde el coatí es una especie muy abundante. Por otro lado, la abundancia alta de $C$. latrans y $D$. virginiana está relacionada posiblemente con sus hábitos alimenticios, ya que estos consumen varios grupos de alimento (omnívoras), las cuales pueden obtener alimento fácilmente (Mesa-Zavala, 2012; Pina, Gómez, \& González, 2004).

El hecho de haber registrado una baja abundancia de $T$. mexicana está relacionado con el diseño experimental utilizado, ya que está sesgado al registro de individuos de hábitos terrestres, por lo que especies con hábitos arborícolas, difícilmente se pueden detectar (Aranda, 2012).

Otra especie poco abundante fue $P$. concolor, que es una especie territorial y requiere de grandes extensiones de terreno para poder desarrollar sus actividades (Lira-Torres, \& Naranjo, 2003; Bustamante, 2008). Sin embargo, Monroy-Vilchis et al. (2011) con el mismo método, registraron mayor abundancia relativa de ésta especie en el Estado de México, probablemente porque muestrearon diversos tipos de vegetación, en los cuales puede habitar la especie.

A diferencia de otros métodos, con las cámaras-trampa también se puede obtener información ecológica de las especies, como patrones de actividad, preferencia de hábitat y estado reproductivo de las mismas (MonroyVilchis et al., 2011; Lira-Torres, \& BrionesSalas, 2012). VanSchaik, \& Griffiths (1996) reportaron una relación entre el tamaño corporal y los patrones de actividad, donde los pequeños mamíferos tienden a ser nocturnos como una estrategia de lucha contra la depredación, esto corresponde con lo obtenido en este estudio en donde, $D$. virginiana y $D$. novemcinctus registraron dicho patrón. Mientras que especies de mayor tamaño registraron un patrón catemeral y diurno.

Los patrones de actividad de P. tajacu corresponden con lo reportado por MonroyVilchis et al. (2011), mencionan que la especie está activa principalmente durante el día; sin embargo, también presentó actividad durante la noche. Además se pudo observar la presencia de crías en los meses de marzo, mayo, agosto y octubre, esto corresponde con lo mencionado por Mayor-Aparicio (2004) quien señala que la especie tiene de 2 a 3 partos por año debido a la rápida gestación que presenta. El tejón $N$. narica, presentó actividad principalmente en el día, estos resultados difieren con lo reportado por Monroy Vilchis et al. (2011). Estos autores mencionan que la especie se mantiene activa las 24 horas del día, con un aumento de actividad entre las 18:00 y las 20:00 horas, esto posiblemente está relacionado a que la especie se alimenta durante el día.

La actividad de L. pardalis sin un horario definido, tampoco corresponde con lo reportado por otros autores (Ayala, Viscarra, \& Wallace, 2010; Díaz-Pulido, \& Payán-Garrido, 2011), ya que esta especie se ha reportado con actividad exclusivamente nocturna. Por el contrario, la actividad catemeral registrada de $C$. latrans, coincide con lo observado en un bosque mesófilo de montaña del estado de Jalisco, México (González-Pérez, Sánchez-Bernal, Íñiguez, \& Santana, 1992). Los patrones de actividad de las dos especies anteriores, apoya lo sugerido por Van Schaik \& Griffiths (1996), quienes mencionan que los animales más grandes 
forrajean tanto de día como de noche debido a sus requerimientos alimenticios.

A través del fototrampeo, se pudieron obtener 22 registros independientes de $D$. novemcinctus, principalmente durante la noche, los resultados coinciden con lo mencionado por Mac Bee \& Baker (1982), quienes mencionan que esta especie es exclusivamente nocturna. En estudios anteriores no se había determinado el patrón de actividad del armadillo por medio de fototrampeo, debido a que dicha técnica no es la adecuada para analizar su abundancia y patrones de actividad (Weckel, Giuliano, \& Silver, 2006; Harmsen, Foster, Silver, Ostro, \& Doncaster, 2010; Monroy-Vilchis et al., 2011). Sin embargo, es posible utilizar cámaras trampa para la evaluación de su abundancia, pero es necesario tomar en cuenta el ámbito hogareño de la especie, para tener un diseño de muestreo adecuado.

Los patrones de actividad de $D$. virginiana han sido reportados como nocturnos (MacManus, 1974; Emmons, \& Feer, 1990), además al ser una especie de menor tamaño tienen mayor riesgo de ser depredada por lo cual, utiliza la oscuridad para no ser detectada fácilmente (Van Schaik, \& Griffiths, 1996).

La presencia de carnívoros como $P$. concolor, $P$. onca y $L$. pardalis, sugiere que la selva comprendida dentro del área de estudio se encuentra en buen estado de conservación, ya que al ser estos, animales depredadores de gran tamaño, necesitan amplios requerimientos de hábitat para poder desarrollarse (Holden, \& Neang, 2009; Cueva, Morales, Brown, \& Peck, 2010). En agosto 2013 se registró la presencia de un individuo joven de $P$. onca por medio de cámaras trampa, dicha especie no había sido reportada anteriormente (Cortés-Marcial, 2009), por lo que el sitio al parecer puede ofrecer las condiciones necesarias para mantener a este depredador (Rumiz et al., 2002).

El bosque tropical caducifolio o selva seca, es un sitio con un alto grado de endemismos y riqueza de especies en México (Ceballos, \& Valenzuela, 2010; Lott, \& Atkinson, 2010); sin embargo, sus componentes florísticos y faunísticos como es el caso de los mamíferos medianos y grandes están en riesgo, debido principalmente a la deforestación para el establecimiento de potreros y áreas de cultivo. Como una estrategia viable en la conservación de estos sitios y de sus componentes, las comunidades indígenas de Oaxaca han establecido "áreas comunales protegidas" como la que se asienta en el área de estudio (Ojo de Agua Tolistoque y Sierra Tolistoque), que han contribuido de manera significativa a la conservación de los mamíferos que ahí habitan. Los datos aquí presentados, servirán para que estas iniciativas tengan mayor sustento y puedan apoyar programas estatales y federales.

\section{AGRADECIMIENTOS}

A las autoridades de las localidades de $\mathrm{La}$ Venta y La Ventosa de Juchitán, Oaxaca por los permisos otorgados para el trabajo de campo. Al Instituto Politécnico Nacional por financiar parcialmente el proyecto a través de los proyectos SIP: 20070443, 20110547, 20121142. Al Consejo Nacional de Ciencia y Tecnología por la beca (229473) otorgada al primer autor. M. B-S agradece a la Secretaría de Investigación y Posgrado, al Sistema de Becas de exclusividad (COFAA) y al de Estímulos al Desempeño a la Investigación (EDI) del Instituto Politécnico Nacional por el apoyo económico. De igual forma al Sistema Nacional de Investigadores por el reconocimiento.

\section{RESUMEN}

Para asegurar la conservación y buen manejo de mamíferos medianos y grandes, es necesario generar información sobre la estructura de la comunidad y de sus poblaciones. El objetivo del presente estudio fue estimar la diversidad, abundancia relativa y patrones de actividad de mamíferos medianos y grandes en una selva baja caducifolia en el Istmo de Tehuantepec, Oaxaca, México. El muestreo se llevó a cabo a intervalos de tres meses de septiembre 2011 a mayo 2013. Se utilizaron dos métodos complementarios: se colocaron 12 cámaras trampa cubriendo un área de $60 \mathrm{~km}^{2}$ y se realizó la búsqueda de rastros en transectos lineales de tres kilómetros de longitud. Se obtuvieron un total de 344 fotografías con un esfuerzo de muestreo de 5292 días-trampa; además, 187 rastros en un total de $144 \mathrm{~km}$ recorridos. En total se registraron 21 
especies de mamíferos, de 13 familias y siete órdenes. El índice de diversidad de Shannon-Wiener fue más alto con el método de rastros $\left(H^{\prime}=2.41\right)$. Las especies más abundantes por medio de rastros fueron: Urocyon cinereoargenteus (IAR=0.23) y Pecari tajacu $(\mathrm{IAR}=0.20)$. Por medio del fototrampeo las especies más abundantes fueron: $P$. tajacu (IAR=2.62) y Nasua narica (IAR=1.28). Los patrones de actividad de $P$. tajacu, N. narica y Odocoileus virginianus fueron diurnos; Canis latrans y Leopardus pardalis no presentaron preferencia hacia algún horario en particular y Didelphis virginiana y Dasypus novemcinctus tuvieron actividad nocturna. Esta información puede ser de ayuda para la creación de programas de manejo y conservación de mamíferos medianos y grandes en el Istmo de Tehuantepec, Oaxaca, México.

Palabras clave: cámaras trampa, rastros, mamíferos, diversidad, patrones de actividad.

\section{REFERENCIAS}

Ahumada, J., Silva, C. E., Gajapersad, K., Hallam, C., Hurtado, J., Martin, E., McWilliam, A., Mugerwa, B., O’Brien, T., Rovero, F., Sheil, D., Spironello, W. R., Winarni, N., \& Andelman, S. J. (2011). Community structure and diversity of tropical forest mammals: data from a global camera trap network. Phiosophical Transactions Royal Society B, 366, 2703-2711.

Aranda, M. (2012). Manual para el rastreo de mamiferos silvestres de México. México: Comisión Nacional para el Conocimientos y Uso de la Biodiversidad.

Arispe, R., Venegas, C., \& Rumiz, D. (2008). Abundancia y patrones de actividad del mapache (Procyon cancrivorous) en un bosque Chiquitano de Bolivia. Mastozoología Neotropical, 15(2), 323-333.

Arriaga, L., Espinoza, J. M., Aguilar, C., Martínez, E., Gómez, L., \& Loa, E. (2000). Regiones terrestres prioritarias de México. México: Comisión Nacional para el Conocimiento y uso de la Biodiversidad.

Ávila, R. \& Medellín, R. (2005). Zorrillo pigmeo. In G. Ceballos \& G. Oliva (Coord.), Los mamiferos silvestres de México (pp. 394-395). México: CONABIOFondo de Cultura Económica.

Ayala, G., Viscarra, M. E., \& Wallace, R. (2010). Density and activity patterns of ocelots (Leopardus pardalis) in Río Hondo, Madidi National Park and Integrated Management Natural Area, La Paz, Bolivia. Revista Boliviana de Ecología y Conservación Ambiental, 28, 119-129.

Barragán, F., Lorenzo, C., Morón, A., Briones-Salas, M. A., \& López, S. (2010). Bat and rodent diversity in a fragmented landscape on the Isthmus of Tehuantepec, Oaxaca, México. Tropical Conservation Science, 3, $1-16$.
Bernard, H., Ahmad, A. H., Brodie, J., Giordano, A. J., Lakim, M., Amat, R., Pei Hue, S. K., Khee, L. S., Tuuga, A., Malim, P. T., Lim-Hasegawa, D., Wai, Y. S., \& Sinun, W. (2013). Camera-Trapping survey of mammals in and around Imbak Canyon Conservation Area in Sabah Malaysian Borneo. The Raffles Bulletin of Zoology, 61(2), 861-870.

Bolaños, C. \& Naranjo, J. E. (2001). Abundancia, densidad y distribución de las poblaciones de ungulados en la cuenca del río Lacatún, Chiapas, México. Revista Mexicana de Mastozoología, 5, 45-57.

Briones-Salas, M. A. \& Sánchez-Cordero, V. (2004). Mamíferos. In A. J. García Mendoza, M. J. Ordóñez \& M. A. Briones-Salas (Eds.), Biodiversidad de Oaxaca (pp. 423-47). México: Instituto de Biología, UNAM-Fondo Oaxaqueño para la Conservación de la Naturaleza-World Wildlife Found.

Buenrostro-Silva, A., Gutiérrez, A., \& García-Grajales, J. (2012). Mamíferos del Parque Nacional Lagunas de Chacahua y La Tuza de Monroy, Oaxaca, México. Acta Zoológica Mexicana (nueva serie), 28(1), 56-72.

Bustamante A. (2008). Densidad y uso de hábitat por los felinos en la parte sureste del área de amortiguamiento del Parque Nacional Corcovado, Península de Osa, Costa Rica. (Tesis de Maestría). Instituto Internacional en Conservación y Manejo de Vida Silvestre, Universidad Nacional, Costa Rica.

Carrillo, E., Wong, G., \& Cuarón, A. D. (2000). Monitoring mammal populations in Costa Rican protected areas under different hunting restrictions. Conservation Biology, 14(6), 1580-1591.

Ceballos, G. \& García, A. (1995). Conserving neotropical biodiversity: the role of dry forest in western Mexico. Conservation Biology, 9, 1349-1353.

Ceballos, G. \& Oliva, G. (2005). Los mamíferos silvestres de México. México: CONABIO-Fondo de Cultura Económica.

Ceballos, G. \& Martínez, L. (2010). Mamíferos. In G. Ceballos, L. Martínez, A. García, E. Espinoza, J. Bezaury, \& R. Dirzo (Eds.), Diversidad, amenazas y áreas prioritarias para la conservación de las selvas secas del Pacifico de México (pp. 119-144). México: Fondo de Cultura Económica, CONABIO.

Ceballos, G. \& Valenzuela, D.(2010). Diversidad, ecología y conservación de los vertebrados de Latinoamérica. In G. Ceballos, L. Martínez, A. García, E. Espinoza, J. Bezaury J., \& R. Dirzo (Eds.), Diversidad, amenazas y áreas prioritarias para la conservación de las selvas secas del Pacífico de México (pp. 94-118). México: Fondo de Cultura Económica, CONABIO.

Cervantes, F. \& Yépez, L. (1995). Species richness of mammals from the vicinity of Salina Cruz, Coastal Oaxaca, Mexico. Anales del Instituto de Biología. Serie Zoología, 66(1), 113-122. 
Cooper, J. D., Waser, P. M., Gopurenko, D., Hellgren, E. C., Gabor, T. M., \& Dewoody, J. A. (2010). Measuring sex-biased dispersal in social mammals: comparisons of nuclear and mitochondrial genes in collared peccaries. Journal of Mammalogy, 91, 1413-1424.

Cortés-Marcial, M. (2009). Diversidad de mamiferos medianos y grandes en dos sitios con diferente grado de conservación en La Venta, Juchitán, Oaxaca. (Tesis de Maestría). CIIDIR-OAX. Instituto Politécnico Nacional, Oaxaca, México.

Cueva, X. A., Morales, N., Brown, M., \& Peck, M. (2010). Macro y mesomamíferos de la Reserva Comunitaria Santa Lucía, Pichincha, Ecuador. Boletín Técnico 9, Serie Zoológica, 6, 98-110.

Diaz-Pulido, A. \& Payán-Garrido, E. (2011). Densidad de ocelotes (Leopardus pardalis) en los llanos Colombianos. Mastozoología Neotropical, 18(1), 63-71.

Dirzo, R. \& Miranda, A. (1991). Altered patterns of herbivory and diversity in the forest: A case study of the possible contemporary defaunation. In P. W. Price, T. M. Lewinsohn, G. W. Fernandes, \& W. W. Benson (Eds.), Evolutionary ecology in tropical and temperate regions (pp. 273-291). New York, USA: John Willey \& Sons Inc.

Doan, T. M. (2003). Which methods are most effective for surveying rainforest herpetofauna? Journal of Herpetology, 37, 72-81.

Emmons, L. H. \& Feer, F. (1990). Neotropical rainforest mammals: a field guide. Chicago, Illinois: The University of Chicago Press.

Espartosa, K., Pinptti, B. \& Pardini, R. (2011). Performance of camera trapping and track counts for surveying large mammals in rainforest remnants. Biodiversity Conservation, 20, 2815-2829.

Gaidet-Drapier, N., Frtz, H., Bogarel, M., Renaud, P. C., Poilecot, P., \& Chardonnet, P. (2006).Cost and efficiency of large mammal census techniques: comparison of methods for a participatory approach in a communal area, Zimbabwe. Biology Conservation, $15,735-754$.

García, E. (1988). Modificaciones al sistema de clasificación climática de Kôppen. México: Instituto de Geografía, Universidad Nacional Autónoma de México.

García-Marmolejo, G., Escalona-Segura, G., \& Van derWal, H. (2008). Multicriteria evaluation of Wildlife management Units in Campeche, Mexico. Journal of wildlife Management, 72(5), 1194-1202.

González, G., Briones-Salas, M. A., \& Alfaro, A. M. (2004). Integración del conocimiento faunístico del estado. In A. J. García Mendoza, M. J. Ordóñez, \& M. A. Briones-Salas (Eds.), Biodiversidad de Oaxaca (pp. 349-366). México: Instituto de Biología, UNAM-Fondo Oaxaqueño para la Conservación de la Naturaleza-World Wildlife Found.
González-Pérez, E., Sánchez-Bernal, V. M., Íñiguez, I., \& Santana, E. (1992). Patrones de actividad del coyote (Canis latrans), la zorra gris (Urocyon cinereoargenteus) y el tlacuache (Didelphis virginiana) en la Sierra de Manantlán, Jalisco. Anales del Instituto de Biología, Universidad Nacional Autónoma de México, Serie Zoología, 63, 293-299.

Goodwin, G. (1969). Mammals from the State of Oaxaca, Mexico in the American Museum of Natural History. Bulletin of the American Museum of Natural History, 141, 1-269.

Guzmán-Lenis, A. \& Camargo-Sanabria, A. (2004). Importancia de los rastros para la caracterización del uso de hábitat de mamíferos medianos y grandes en el bosque Los Mangos (Puerto López, Meta, Colombia). Acta Biológica Colombiana, 9(1), 11-22.

Hall, E.R. (1981). The Mammals of North America. New York: The Ronald Press Co.

Harmsen, B. J., Foster, R. J., Silver, S., Ostro, L., \& Doncaster, C. P. (2010). Differential use of trails by forest mammals and the implications for camera-trap studies: a case study from Belize. Biotropica, 42, 126-133.

Hernández, I. U., Ellis, E. A., \& Gallo, C. A. (2013). Aplicación de teledetección y sistemas de información geográfica para el análisis de deforestación y deterioro de selvas tropicales en la región Uxpanapa, Veracruz. Geo Focus (Informes y Aplicaciones), 13, 1-24.

Holden, J. \& Neang, T. (2009). Small carnivore records from the Cardamom Mountains, southwestern Cambodia. Small Carnivore Conservation, 40, 16-21.

IUCN (International Union for Conservation of Nature). (2011). Red list of threatened species. www.iucnredlist.org (consultado en octubre 2013).

Lira, I. \& Naranjo, E. (2003). Abundancia, preferencia de hábitat e impacto del ecoturismo sobre el puma y dos de sus presas en la Reserva de la Biosfera El Triunfo, Chiapas, México. Revista mexicana de Mastozoología, 7, 20-39.

Lira-Torres, I. \& Briones-Salas, M. A. (2012). Abundancia relativa y patrones de actividad de los mamíferos de los Chimalapas, Oaxaca, México. Acta Zoológica Mexicana (ns), 28(3), 566-585.

Logan, C. J. \& Longino, J. T. (2013). Adult male coatis play with a band of juveniles. Brazilian Journal of Biology, 73, 353-355.

Lott, E. \& Atkinson, T. H. (2010). Diversidad florística. In G. Ceballos, L. Martínez, A. García, E. Espinoza, J. Bezaury, \& R. Dirzo (Eds.), Diversidad, amenazas y áreas prioritarias para la conservación de las selvas secas del Pacífico de México (pp. 63-76). México: Fondo de Cultura Económica, CONABIO.

Luis, A. M., Llorente, J. B., Warren, A. D., \& Vargas, I. F. (2004). Los lepidópteros: papilionoideos y 
hesperioideos. In A. J. García Mendoza, M. J. Ordóñez, \& M. A. Briones-Salas (Eds.), Biodiversidad de Oaxaca (pp. 331-356). México: Instituto de Biología, UNAM-Fondo Oaxaqueño para la Conservación de la Naturaleza-World Wildlife Found.

Lyra-Jorge, M. C., Ciocheti, G., Pivello, V. R., \& Meirelles, S. T. (2008). Comparing methods for sampling large-and medium-sized mammals: camera traps and track plots. European Journal of Wildlife Research, 54(4), 739-744.

Mac Bee, K. \& Baker, R. (1982). Dasypus novemcinctus. Mammalian Species, 162, 1-9.

Maffei, L., Cuellar, E., \& Noss, J. (2002). Uso de trampascámara para la evaluación de mamíferos en el ecotono Chaco-Chiquitanía. Revista Boliviana de Ecología y Conservación Ambiental, 11, 55-65.

Maffei, L., Noss, A. J., \& Fiorello, C. (2007). The jaguarundi (Puma yagouaroundi) in the kaa-iya del gran Chaco National Park, Santa Cruz, Bolivia. Mastozoología Neotropical, 14(2), 263-266.

Magurran, A. E. (1988). Ecological diversity and its measurement. New Jersey: Princeton University Press.

Marnewick, K., Funston, P. J., \& Karanth, K. U. (2008). Evaluating camera trapping as a method for estimating cheetah abundance in ranching areas. South African Journal of Wildlife Research, 38(1), 59-65.

Martin, G., Camacho, C. I., Del Campo, C. A., Anta, S., Chapela, F., \& González, M. A. (2011). Indigenous and community conserved areas in Oaxaca, Mexico. Management of Environmental Quality, 22(2), 250-266.

Mayor-Aparicio, P. G. (2004). Fisiología reproductiva y desarrollo de métodos diagnósticos del estado reproductivo de la hembra pecari de collar (Tayassu tajacu, Linnaeus 1758) de la Amazonia. (Tesis doctoral). Universidad Autónoma de Barcelona, España.

McManus, J. (1974). Didelphis virginiana. Mammalian Species, 40, 1-6.

Medellín, R., Azuara, D., Maffei, L., Zarza, H., Bárcenas, H., Cruz, E., Legaria, R., Lira, I., Ramos-Fernández, G., \& Ávila, S. (2006). Censos y Monitoreo. In C. Chávez \& G. Ceballos (Eds.). El Jaguar Mexicano en el Siglo XXI: Situación Actual y Manejo (pp. 25-35). México: CONABIO-ALIANZA WWF TELCELUniversidad Nacional Autónoma de México.

Mesa-Zavala, E., Álvarez-Cárdenas, S., Galina-Tessaro, P., Troyo-Diéguez, E., \& Guerrero-Cárdenas, I. (2012). Vertebrados terrestres registrados mediante fototrampeo en arroyos estacionales y cañadas con agua superficial en un hábitat semiárido de Baja California Sur, México. Revista Mexicana de Biodiversidad, 83(1), 235-245.

Michalski, F. \& Peres, C. A. (2007). DisturbanceMediated mammal abundance-area relationships in
Amazonian forest fragments. Conservation Biology, $21,1626-1640$

Monroy-Vilchis, O., Zarco-González, M. M., RodríguezSoto, C., Soria-Díaz, L., \& Urios V. (2011). Fototrampeo de mamíferos, en la Sierra Nanchititla, México: abundancia relativa y patrón de actividad. Revista de Biologia Tropical, 59 (1), 373-383.

Moreno, C. E. \& Halffter, G. (2000). Assessing the completeness of bat biodiversity inventories using species accumulation curves. Journal Applied Ecology, 37, 149-158.

Moreno, C. (2001). Métodos para medir la biodiversidad, vol. 1. M \& T-Manuales y Tesis SEA, Zaragoza.

Munari, D., Keller, C., \& Venticinque, E. (2011). An evaluation of field techniques for monitoring terrestrial mammal populations in Amazonia. Mammal Biology, 76, 401-408

Nakashima, Y., Inoue, E., Inoue-Murayama, M., \&. Sukor, J. R. A. (2010). Functional uniqueness of a small carnivore as seed dispersal agents: A case study of the common palm civets in the Tabin Wildlife Reserve, Sabah, Malaysia. Oecologia, 164, 721-730.

Naranjo, E., \& Cuarón, A. (2010). Uso de la fauna silvestre. In G. Ceballos, L. Martínez, A. García, E. Espinoza, J. Bezaury, \& R. Dirzo (Eds.), Diversidad, amenazas $y$ áreas prioritarias para la conservación de las selvas secas del Pacífico de México (pp. 271-283). México: Fondo de Cultura Económica, CONABIO.

Navarro, S. A., García-Trejo, E. A., Peterson, A. T., \& Rodríguez-Contreras, V. (2004). Aves. In A. J. García Mendoza, M. J. Ordóñez, \& M. A. Briones-Salas (Eds.), Biodiversidad de Oaxaca (pp. 391-421). México: Instituto de Biología, UNAM-Fondo Oaxaqueño para la Conservación de la Naturaleza-World Wildlife Found.

O’Brien, T. G., Kinnaird, M. F., \& Wibisono, H. T. (2003). Crouching tigers, hidden prey: Sumatran tiger and prey populations in a tropical forest landscape. Animal Conservation, 6, 131-139.

Odell, E. A. \& Knight, R. L. (2001). Songbird and mediumsized mammal communities associated with exurban development in Pitkin County, Colorado. Conservation Biology, 5(4), 143-1150.

Olguin Monroy, H., León, L., Samper-Palacios, U. M., \& Sánchez-Cordero, V. (2008). Mastofauna de la región de los Chimalapas, Oaxaca, México. In C. Lorenzo, E. Espinoza, \& J. Ortega (Eds.), Avances en el estudio de los mamíferos II. Publicaciones Especiales Vol. II (pp. 165-216). México: Asociación Mexicana de Mastozoología, A.C.

Olson, D., Dinerstein, E., Abell, R., Allnett, T., Carpenter, C., McClenachan, L., D’Amico, J., Hurley, P., Kassem, K., Strand, H., Taye, M., \& Thieme, M. (2000). The Global 200: A representation approach 
to conserving the earth's distinctive ecoregions. World Wildlife Funds.

Orr, M. \&. Smith, T. B. (1998). Ecology and speciation. Tree, 13(12), 502-506.

Ortega, D., Sánchez, G., Solano, C., Huerta, M. A., Meza, V., Romero, J., Cruz, L., Palacios, T., Montes, E., \& Galindo-Leal, C. (2010). Áreas de Conservación Certificadas en el Estado de Oaxaca. México: WWFCONANP Oaxaca, México.

Ortíz-Martínez, T. \& Rico-Gray, V. (2007). Spider monckeys (Ateles geoffroyi vellerosus) in a tropical decidous forest in Tehuantepec, Oaxaca, México. The Southwestern Naturalist, 52, 393-399.

Ortíz-Pérez, M., Hernández, J. R., \& Figueroa, J. M. (2004). Reconocimiento fisiográfico y geomorfológico. In A. J. García Mendoza, M. J. Ordóñez, \& M. A. Briones-Salas (Eds.), Biodiversidad de Oaxaca (pp. 43-54). México: Instituto de Biología, UNAM-Fondo Oaxaqueño para la Conservación de la NaturalezaWorld Wildlife Found.

Pérez-García, E. A., Meave, J., \& Gallardo, C. (2001). Vegetación y flora de la región de Nizanda, Istmo de Tehuantepec, Oaxaca. Acta Botánica Mexicana, $56,19-88$.

Pérez-García, E., Meave, J., \& Salas, S. (2010). Nizanda, Oaxaca. In G. Ceballos, L. Martínez, A. García, E. Espinoza, J. Bezaury, \& R. Dirzo (Eds.), Diversidad, amenazas y áreas prioritarias para la conservación de las selvas secas del Pacífico de México (pp. 538-542). México: Fondo de Cultura Económica, CONABIO.

Pérez-Irineo, G. \& Santos-Moreno, A. (2012). Diversidad de mamíferos de talla grande y media de una selva subcaducifolia del noreste de Oaxaca, México. Revista Mexicana de Biodiversidad, 83,164-169.

Peterson, A. T., Soberón, J., \& Sánchez-Cordero, V. (1999). Conservatism of ecological niches in evolutionary time. Science, 285, 1265-1267.

Pina, G. P. L., Gómez, R. A. C., \& González, C. A. L. (2004). Distribution, habitat association and activity patterns of medium and large sized mammals of Sonora, Mexico. Natural Areas Journal, 24, 354-357.

Ramamoorthy, T. P., Bye, R., Lot, A., \& Fa, J. (1998). Diversidad biológica de México: Orígenes y distribución. México: Instituto de Biología-Universidad Nacional Autónoma de México.

Reid, A. F. (1997). A Field guide to the mammals of Central and Southeast Mexico. New York: Oxford University Press.

Robinson, J. G., \& Bennett, E. L. (2000). Hunting for Sustainability in Tropical Forests. New York: Columbia University Press.

Rumiz, D., Fuentes, A., Rivero, K., Santibáñez, J., Cuellar, E., Miserendino, R., Fernández, I., Maffei, L., \&
Taber, A. (2002). La biodiversidad de la Estancia San Miguelito, Santa Cruz-Bolivia: Una justificación para establecer reservas privadas de conservación. Instituto de Ecología, La Paz, Bolivia. Documentos Ecología en Bolivia, Serie Biodiversidad, 1, 1-67.

Ryan, T. J., Philippi, T., Leiden, Y. A., Dorcas, M. E., Wigley, T. B., \& Gobbons, J. W. (2002). Monitoring herpetofauna in a managed forest landscape: effects of habitat types and census techniques. Forest Ecology and Management, 167, 83-90.

Rzedowski, J. (1978). Vegetación de México. México: Limusa. México.

Santos-Moreno, A. \& Ruíz-Velásquez, E. (2011). Diversidad de mamíferos de la región de Nizanda, Juchitán, Oaxaca, México. Therya, 2(2), 155-168.

Secretaría de Medio Ambiente, Recursos Naturales (SEMARNAT). (2010). Norma Oficial Mexicana NOM-059-ECOL-2010. Protección ambiental, especies de flora y fauna silvestres de México, categorías de riesgo y especificaciones para su inclusión, exclusión o cambio, y lista de especies en riesgo. Diario Oficial de la Federación, Jueves 30 de diciembre de 2010, 1, 1-77.

Shek, C. T., Chan, C. S. M., \& Wan, Y. F. (2007). Camera trap survey of Hong Kong terrestrial mammals in 2002-06. Biodiversity, 15, 1-11.

Simonetti, J. A. \& Huareco, I. (1999). Uso de huellas para determinar diversidad de mamíferos en la Reserva de la Biósfera Estación Biológica Beni, Bolivia. Mastozoología Neotropical, 6, 139-144.

Swan, M., Di Stefano, J., Chistie, F., Steel, E., \& York, A. (2014). Detecting mammals in heterogeneous landscapes: implications for biodiversity monitoring and management. Biodiversity and Conservation, 23, 343-355.

Tobler, M. W., Carrillo-Percastegui, S. E., \& Powell, G. (2009). Habitat use, activity patterns and use of mineral licks by five species of ungulate in South-Eastern Peru. Journal of Tropical Ecology, 25, 261-270.

Van Schaik, C. P. \& Griffiths, M. (1996). Activity periods of Indonesian rain forest mammals. Biotropica, 28, 105-112.

Varma, S., Pittet, A., \& Jamadagni, H. S. (2006). Experimenting usage of camera-traps for population dynamics study of the Asian elephant Elephas maximus in southern India. Current Science, 91(3), 324-331.

Weckel, M., Giuliano, W., \& Silver, S. (2006). Jaguar (Panthera onca) feeding ecology: distribution of predator and prey through time and space. Journal of Zoology, 270, 25-30.

Whittaker, R. H. (1972). Evolution and measurement of species diversity. Taxon, 21(2/3), 213-251.

Wilson, G. J. \& Delahay, R. J. (2001). A review of methods to estimate the abundance of terrestrial carnivores 
using field signs and observation. Wildlife Research, 28, 151-164.

Yasuda, M. (2004). Monitoring diversity and abundance of mammals with camera traps: a case study on Mount Tsukuba, central Japan. Mammal Study, 29, 37-46.

Zar, J. (1999). Biostatistical analysis (Fourth Edition). USA: Prentice Hall.
Zielinski, W. J. \& Kucera T. E. (1995). Survey methods for the detection of wolverines, Lynx, fishers and martens (Report PSW-157). USDA Forest Service General Technical. 